\title{
In Vitro Reconstitution of EryClII Activity for the Preparation of Unnatural Macrolides
}

\author{
Yanqiu Yuan $\ddagger, \dagger$, Hak Suk Chung $\ddagger$, Catherine Leimkuhler $\ddagger$, Christopher T. Walsh§, Daniel \\ Kahne $\neq, \S$, and Suzanne Walker $\neq, \uparrow$ \\ $\dagger$ Department of Microbiology and Genetics, Harvard Medical School, Boston, Massachusetts 02115 \\ $\S$ Department of Biological Chemistry and Molecular Pharmacology, Harvard Medical School, Boston, \\ Massachusetts 02115 \\ $\$$ Department of Chemistry and Chemical Biology, Harvard University, Cambridge, Massachusetts 02138
}

Macrolides and anthracyclines are clinically important drugs produced by streptomycetes. Both classes of natural products contain aminosugar moieties that are attached by glycosyltransferases (Gtfs) at a late stage in the biosynthetic pathway and are essential for biological activity (Scheme 1). ${ }^{1}$ These Gtfs are potentially useful for the combinatorial biosynthesis or chemoenzymatic synthesis of structurally novel compounds with new biological activities. ${ }^{2}$ For many years, however, all attempts to reconstitute the activity of these Gtfs in vitro failed. Then, in 2004, Liu and coworkers reported that DesVII, a TDP-Ddesosaminyl tranferase involved in pikromycin biosynthesis, requires the presence of an "auxiliary protein", DesVIII, encoded in the same gene cluster, to catalyze glycosyltransfer in vitro, although the role of DesVIII was not clear. ${ }^{3}$ Walsh and coworkers subsequently reported that AknS, a Gtf involved in the glycosylation of aklavinone, requires the presence of the DesVIII homolog, AknT, to effect glycosyltransfer in vitro. ${ }^{4}$ Walsh and coworkers proposed that AknT functions as a regulatory subunit that increases the catalytic activity of AknS by influencing substrate affinity. ${ }^{4}$ There is precedent for this hypothesis because a wellcharacterized eukaryotic glycosyltransferase, $\beta$-1,4-galactosyltransferase, contains a regulatory subunit that alters substrate affinity and selectivity. ${ }^{5}$

It is important to understand the role of Gtf auxiliary proteins in order to facilitate the use of aminosugar transferases in the chemoenzymatic synthesis of macrolide and anthracycline analogs. Therefore, we have been studying another aminosugar transferase, EryCIII, which is involved in erythromycin biosynthesis (Scheme 1B). ${ }^{6,7}$ EryCIII catalyzes the transfer of Ddesosamine to 3- $\alpha$-mycarosylerthronolide $\mathrm{B}(\alpha \mathrm{MEB})$, making erythromycin $\mathrm{D}(\mathrm{EryD}, 4)$. We previously reported that EryCIII can be expressed in active form in E. coli provided that the GroEL/ES chaperonin complex is coexpressed. ${ }^{6}$ However, the purified protein was unstable and lost activity within hours of isolation 6,8 and attempts to stabilize the activity failed. Because the erythromycin gene cluster encodes a DesVIII homolog, EryCII, we sought to determine whether EryCII influenced the stability or activity of EryCIII. Studies described below show that EryCII does activate EryCIII. Surprisingly, however, we found that EryCIII remains active when EryCII is removed, which rules out models for EryCIII activation that involve a stable EryCII:EryCIII complex during glycosyltransfer. We also found that the noncognate auxiliary protein AknT stably activates EryCIII. Moreover, activated EryCIII is

E-mail: suzanne_walker@hms.harvard.edu.

Supporting Information Available: Experimental procedures and data for kinetics; MS characterization of products; synthesis and characterization of TDP-sugars. This material is available free of charge via the Internet http://pubs.acs.org. 
capable of utilizing an unnatural sugar donor, enabling the preparation of erythromycin analogs. Thus, this work shows how to reconstitute the activity of EryCIII in vitro for the synthesis of unnatural macrolides.

We PCR amplified eryCII from a mutant strain of Saccharopolyspora erythraea NRRL 2338, $\triangle e r y C I-60^{9}$ and cloned the gene into a pET22b expression vector to obtain the plasmid pERY2, which was then transformed into $E$. coli BL21(DE3). Expressed as a C-terminal His ${ }_{6}$ fusion protein, EryCII was partially purified via Ni-NTA affinity chromatography and then added to a reaction mixture containing inactive EryCIII expressed as previously described. ${ }^{6}$ EryCIII treated with EryCII mixture was found to be catalytically active, effecting transfer of desosamine to $\alpha \mathrm{MEB}$. The untreated EryCIII sample showed no activity.

We then examined whether AknT, shown to be required for AknS activity in the aclarubicin pathway, can activate purified, inactive EryCIII. AknT is 26\% homologous to EryCII and AknS is $48 \%$ homologous to EryCIII. To our surprise, we found that the addition of purified AknT to inactive EryCIII restores the activity of EryCIII in vitro (Figure 2C)..$^{10}$ Thus, both EryCII and AknT can function as glycosyltransferase activator proteins for EryCIII, although we note that larger amounts of AknT are required to achieve the same level of activation (Figure 2C). Given the structural differences in the substrates of EryCIII and AknS, this result rules out models for activation that involve binding of substrates by the auxiliary proteins.

To evaluate whether EryCII forms a stable complex with EryCIII during the glycosyltransfer reaction, we prepared a plasmid encoding both native EryCII and C-terminal His 6 -tagged EryCIII. Protein expression was induced with IPTG and EryCIII was purified away from EryCII by Ni-NTA affinity chromatography. (We designate the coexpressed, purified enzyme as EryCIII*.) No EryCII was visible in a SDS-PAGE gel of the purified EryCIII* sample, and mass spectral analysis showed that it contained less than 2\% EryCII. Nevertheless, EryCIII* was not only fully active (Figure 2D), but it retained activity for at least two months under a range of different storage conditions.

The above experiments suggest that once EryCIII is activated by EryCII, EryCII is no longer required. To assess the generality of this observation, we treated inactive EryCIII with native AknT for 3 hours, and then purified EryCIII by Ni-NTA affinity chromatography to remove AknT. This EryCIII sample, like EryCIII*, proved to have stable enzymatic activity. Although we cannot rule out the possibility that minute amounts of auxiliary protein influence enzymatic activity, we note that relatively high concentrations of other auxiliary proteins $(0.25-3$ equivalents) seem to be required to effect glycosyltransfer. ${ }^{3,4}$

Having identified conditions to obtain pure, active EryCIII, we were able to characterize the kinetic behavior of the enzyme. The apparent $K_{m}$ of EryCIII* for $\alpha \mathrm{MEB}$ (at $1 \mathrm{mM}$ TDP-Ddesosamine) was found to be $50 \mu \mathrm{M}$ and the apparent $k_{c a t}$ was $1 \mathrm{~min}^{-1}$ (Supporting Information), which is comparable to the turnover numbers observed for many other antibiotic Gtfs. We also investigated the substrate selectivity of the purified sample and found that Dmycaminose can be transferred to the aMEB aglycone (3) after an overnight incubation to produce the corresponding mycaminosyl erythromycin D derivative, erythromycin M (EryM, 5) (Figure 3). ${ }^{6}$ Thus, the activated enzyme is capable of transferring an alternative sugar donor. This is the first report of EryCIII having some substrate flexibility for alternative sugar donors. 11 Because the desosamine sugar contacts the 50S subunit of ribosome, ${ }^{12}$ the discovery that an unnatural sugar can be transferred has implications for the synthesis of new erythromycin derivatives with improved properties.

In conclusion, we have shown that EryCIII can be activated by both EryCII and the non-cognate Gtf auxiliary protein AknT. We can remove $>98 \%$ of the auxiliary proteins without any apparent loss of activity, which suggest that these proteins do not function as a regulatory 
subunit during glycosyltransfer reaction. Given that EryCIII can be transiently recovered in active form when expressed with GroEL/ES, ${ }^{6}$ and that including high concentrations of glycerol (>15\%), known to facilitate folding, restores partial activity to the enzyme, ${ }^{13}$ we propose that EryCII or AknT facilitates a conformational change of EryCIII from an inactive to an active form.

We are currently trying to understand in greater detail the mechanism by which pre-exposure to EryCII or AknT activates EryCIII for subsequent glycosyltransfer. In the meantime, this report sets the stage for more detailed investigations of the substrate selectivity of EryCIII, which could lead to new antibiotics with activity against resistant microorganisms.

\section{Supplementary Material}

Refer to Web version on PubMed Central for supplementary material.

\section{Acknowledgement}

This research was supported by the NIH (A144854 to S.W., GM66174 to D.K. and GM20011 to C.T.W.). We thank Kosan Biosciences for providing a gift of $\alpha \mathrm{MEB}$.

\section{References}

1. a Weymouth-Wilson AC. Nat. Prod. Rep 1997;14:99-110. [PubMed: 9149408] b Thorson JS, Hosted JTJ, Jiang J, Biggins JB, Ahlert J. J. Curr. Org. Chem 2001;5:139-167.

2. a Katz L, Ashley GW. Chem. Rev 2005;105:499-528. [PubMed: 15700954] b Oberthur M, Leimkuhler C, Kruger RG, Lu W, Walsh CT, Kahne D. J. Am. Chem. Soc 2005;127:10747-10752. [PubMed: 16045364]

3. Borisova SA, Zhao LS, Melancon CE, Kao CL, Liu HW. J. Am. Chem. Soc 2004;126:6534-6535. [PubMed: 15161264]

4. Lu W, Leimkuhler C, Gatto G, Kruger R, Oberthur M, Kahne D, Walsh C. Chem. Biol 2005;12:527534. [PubMed: 15911373]

5. Ramakrishnan B, Boeggeman E, Ramasamy V, Qasba PK. Curr. Opin. Struct. Biol 2004;14:593-600. [PubMed: 15465321]

6. Lee HY, Chung HS, Hang C, Khosla C, Walsh CT, Kahne D, Walker S. J. Am. Chem. Soc 2004;126:9924-9925. [PubMed: 15303858]

7. a Gaisser S, Bohm GA, Cortes J, Leadlay PF. Mol. Gen. Genet 1997;256:239-251. [PubMed: 9393448] b Summers RG, Donadio S, Staver MJ, Wendt-Pienkowski E, Hutchinson CR, Katz L. Microbiology 1997;143:3251-3262. [PubMed: 9353926]

8. We were unable to remove GroEL completely from the purified protein.

9. Vara J, Lewandowska-Skarbek M, Wang Y, Donadio S, Hutchinson C. J. Bacteriol 1989;171:58725881. [PubMed: 2681144]

10. Heat treatment of $\operatorname{AknT}\left(95^{\circ} \mathrm{C}, 15 \mathrm{~min}\right)$ destroys its ability to activate EryCIII.

11. Mendez C, Salas JA. Trends Biotechnol 2001;19:449-456. [PubMed: 11602309]

12. Schlünzen F, Zarivach R, Harms J, Bashan A, Tocilj A, Albrecht R, Yonath A, Franceschi F. Nature 2001;413:814-821. [PubMed: 11677599]

13. Sawanoa H, Koumotob Y, Ohtab K, Sasakia Y, Segawac S, Tachibana H. FEBS Letters 1992;303:1114. [PubMed: 1592107] 


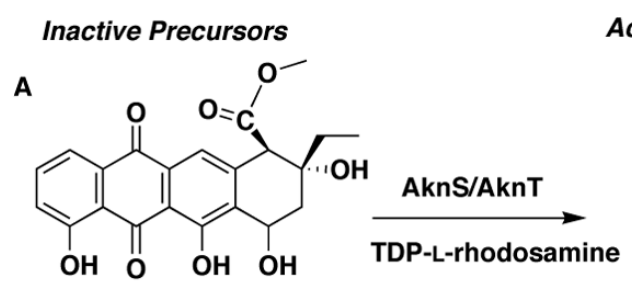

Active Glycoslyated Metabolites

aklavinone (1)

B

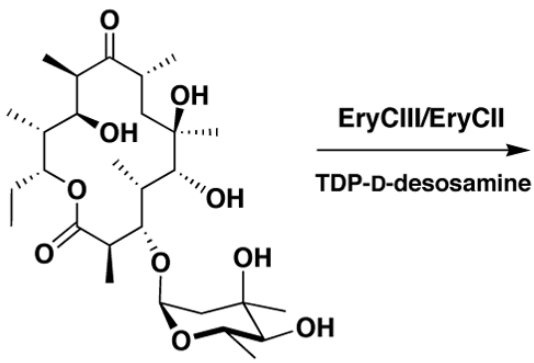

3- $\alpha$-mycarosylerythronolide B (3)

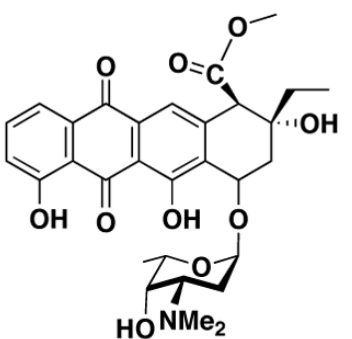

rhodosaminyl-aklavinone (2) precursor of aclarubicin

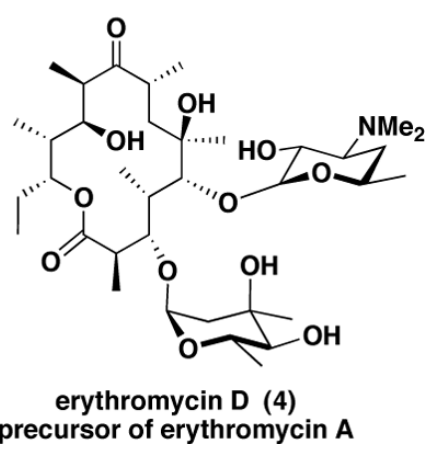

Scheme 1. 

A) Purified, inactive EryCIII + EryCII
or AknT
Active EryCIII
B) EryCIII coexpressed with EryCII
purification
Active EryCIII

Figure 1.

Two Different Ways to Activate EryCIII. A) Addition of either EryCII or AknT to purified, inacitve EryCIII in situ. B) Coexpression of EryCIII with EryCII and subsequent purification away from EryCII. 


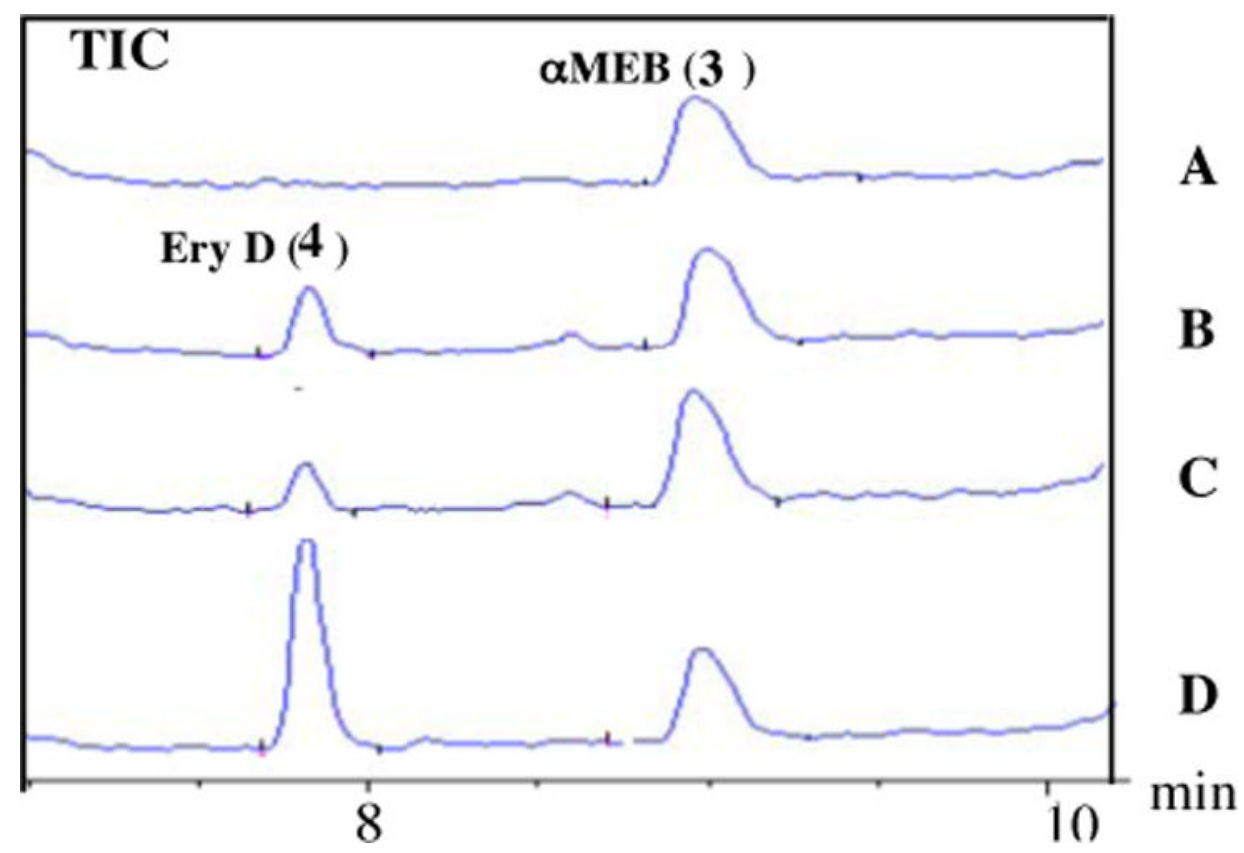

Figure 2.

TIC traces for glycosylation reactions catalyzed by different batches of EryCIII (for detailed reaction conditions, see supporting information). (A) $1.2 \mu \mathrm{M}$ EryCIII; (B) $1.2 \mu \mathrm{M}$ EryCIII, 0.4 $\mu \mathrm{M}$ EryCII; (C) $1.2 \mu \mathrm{M}$ EryCIII, $2.4 \mu \mathrm{M}$ AknT; (D) $1.2 \mu \mathrm{M}$ EryCIII*. 


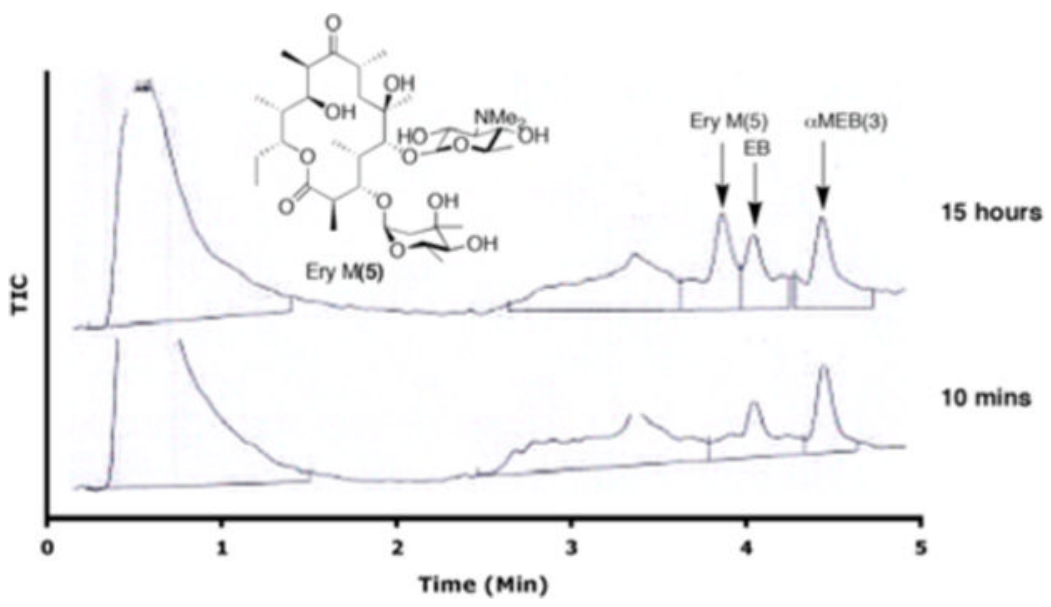

Figure 3.

EryCIII* catalyzed glycosylation of $\alpha \mathrm{MEB} 3$ with TDP-D-mycaminose. Reaction conditions are as in Figure 2 except the sugar donor and the reactions are quenched at the times indicated in the figure $\left([\mathrm{M}+\mathrm{H}]^{+}\right.$calculated 720.45 ; observed 720.5$)$. EB: Erythronolide B is present in the starting material and we have verified that it's not a substrate fro EryCIII. 